\title{
Rare Cause of Cephalalgia in a Young Woman - a Case Report
}

\author{
Varga Andreea, Szakacs Xantus Timea*, Gliga Mirela, Podoleanu Cristian Gheorghe Calin, Bocicor \\ Andreea Elena, Carasca Emilian, Tilea Ioan
}

University of Medicine and Pharmacy Tirgu Mures, Romania

Background: Young adults meeting hypertension diagnostic criteria have a lower prevalence of a hypertension diagnosis. Headache is a rather common symptom among young people. Fibromuscular dysplasia (FMD) is an idiopathic, segmental, nonatherosclerotic and noninflammatory disease of the muscular tunica of arterial walls, leading to stenosis of small and medium-sized arteries. Fibromuscular dysplasia is much more common than previously thought and is a treatable cause of secondary hypertension. Case presentation: We present the case of an 18 y.o. young woman, with headache and high blood pressure. "White coat hypertension" was suspected. Clinical history with abrupt onset and increasingly difficult to treat hypertension especially in women, were suggestive for renal artery stenosis. Renal ultrasound and digital subtraction angiography confirmed the aspect of FMD. Sequential percutaneous renal artery angioplasty was later performed with improved evolution both from the clinical point of view and controlled blood pressure below 140/90 mmHg with minimal antihypertensive regimen. Angio CT exam of neck and brain arteries was performed, no other FMD typical lesions were identified. Conclusions: Medical treatment is first indicated for the hypertensive patient. In this particular case percutaneous renal artery angioplasty showed significant improvement in reduction of antihypertensive treatment in a young patient with secondary hypertension. Further monitoring and management of this patient will include blood pressure measurements at 3-month intervals and renal function measurements annual, as well as non-invasive duplex ultrasonography at 12-month intervals, follow-up is indefinite. It remains challenging whether the patient can be medically managed on antihypertensive medication alone.

Keywords: family medicine, secondary hypertension, fibromuscular dysplasia, treatment

Received: 9 December 2014 / Accepted: 27 April 2015

\section{Introduction}

Headache is a rather common symptom among young people. It can become a health issue in 12 to 18 years old category of patients especially because it affects their quality of life, increases the number of absences in school and it can raise the health-related costs in young population. Headache as a symptom is more frequent in girls, with a higher prevalence range for migraine and episodic tensiontype headache [1].

Fibromuscular dysplasia is an idiopathic, segmental, nonatherosclerotic and non-inflammatory disease of the muscular of arterial walls, leading to stenosis of small and medium-sized arteries [2]. It most commonly involves the renal arteries (60-70\% of cases) followed by cervico-cranial, non-renal abdominal and coronary arteries [3-5]. In about one third of cases it affects multiple vascular beds. Fibromuscular dysplasia is much more common than previously thought, perhaps affecting as many as $4 \%$ of adult women [6]. Compared to middle-aged and older adults, young adults meeting hypertension diagnostic criteria have a lower prevalence of a hypertension diagnosis [7].

\section{Case presentation}

We present the case of an 18-year-old young woman, who presented herself to her family physician office with cephalalgia, fatigue, and palpitations. The main symptom was

* Correspondence to: Timea Szakacs Xantus

E-mail: extimi@yahoo.com an intermittent pulsating headache, with an insidious onset, localised mainly in the forehead, which occurred in different moments of day, independent of exercise, stress, emotions or other triggers. No previous significant diseases in her past medical history, no active or passive smoking and usage of oral contraceptives were noticed. Family history was positive for primary hypertension in both maternal grandparents. The physical examination revealed a body mass index of $20.7 \mathrm{~kg} / \mathrm{m}^{2}$, apparently no respiratory or infectious disease and no signs of neurological disorder at the time of examination. No detectable heart or vascular, including abdominal vascular bruits were detected. Vital signs including blood pressure measurement were assessed, by using a blood pressure device with appropriate size cuff. Blood pressure in left arm was $155 / 90 \mathrm{mmHg}$ and $160 / 90 \mathrm{mmHg}$ in right arm, in sitting position after 5 minutes at rest, with heart rate about $95 \mathrm{bpm}$. At this time "white coat hypertension" was suspected, as the patient had high office blood pressure and no asymptomatic organ damage, with criteria for low total cardiovascular risk. For diagnostic evaluation, out-of-office blood pressure measurement, using home blood pressure monitoring was considered. She was proper trained on the methodology of home blood pressure monitoring in order to reduce errors during measurement and provide reliable assessment of home blood pressure.

The patient returned at the family physician office after one week of home blood pressure measurements, in order to define the appropriate management strategy. Triple an- 
tihypertensive drug treatment was initiated using angiotensin-converting-enzyme inhibitor, diuretic and calcium channel blocker. After 14 days, there was no adequate response to antihypertensive drug therapy, blood pressure values remaining $180 / 100 \mathrm{mmHg}$ and persisting headache.

In order to identify the aetiology of hypertension (suspected secondary hypertension) and maximize prediction of cardiovascular risk the patient was referred in a tertiary hospital. Complete evaluation using routine and additional tests, were performed based on history, physical examination and findings from previous laboratory tests.

Metabolic panel was within normal ranges including unimpaired kidney function. Her 12-leads rest electrocardiography detected normal axis, sinus tachycardia, left ventricular hypertrophy (figure 1).

Echocardiography confirmed the left ventricular hypertrophy, an ejection fraction of $60 \%$, with no signs of left ventricular dysfunction. Clinical history with abrupt onset and increasingly difficult to treat hypertension were suggestive for renal artery stenosis, with suspected fibromuscular dysplasia. Endocrinology evaluation combined with hormonal laboratory findings excluded endocrine secondary hypertension.

A Philips $\mathrm{HD}^{\circ} 1^{\circ}$ ultrasound system was used for the abdominal sonography. Asymmetric kidneys were visualised: left kidney $-9.6 \mathrm{~cm}$ in long axis, comparing to the

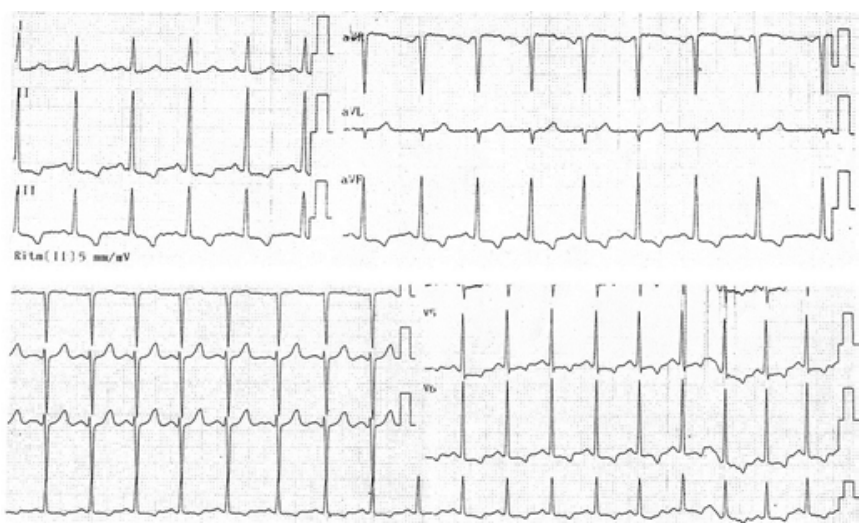

Fig. 1. Rest ECG left ventricular hypertrophy

right kidney that had $10.3 \mathrm{~cm}$. (figure 2, 3).

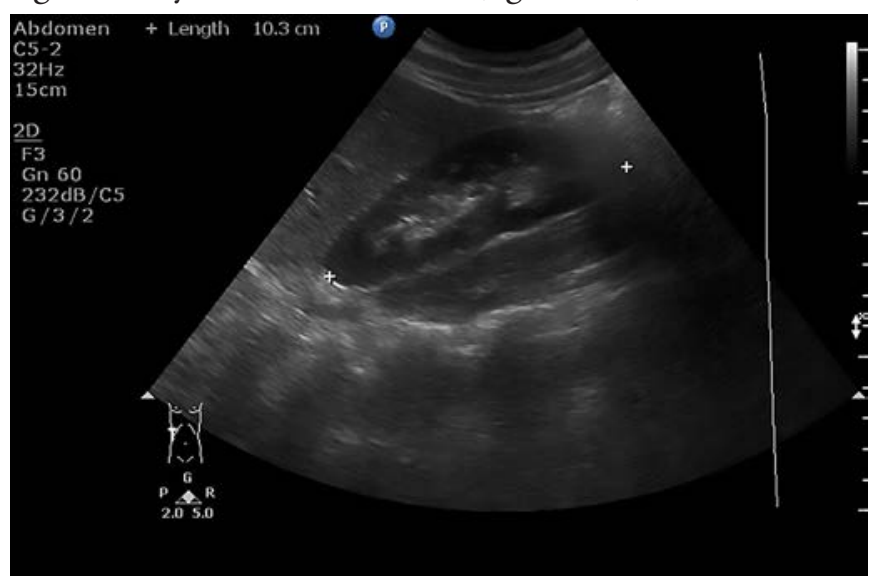

Fig. 2. Right kidney morphology in grey scale
In the colour Doppler examination, the origin of both renal arteries was localized from the aorta and a turbulent flow was noticed in both arteries (figure 4).

High blood velocities were found in both renal arteries, peak systolic velocity being within $140-286 \mathrm{~cm} / \mathrm{sec}$ in consecutive measurements. Renal artery stenosis was suspected (figure 5).

Intrarenal spectral Doppler examination revealed the "parvus and tardus" waveform in the renal segmental and interlobar branches, characteristic for the renal flow distal to a stenosis. (figure 6,7).

The patient was referred to renal angiography with digital subtraction in order to evaluate the renal arteries. A typical „string-of-beads” appearance was identified in both renal arteries, in mid portion, that confirmed the diagnosis of fibromuscular dysplasia (figure 8, 9).

Sequential (right and then left) percutaneous transluminal renal angioplasty (PTRA) using standard technique was performed with good results (figure 10, 11).

Patient was discharged a few days later with only one anti-hypertensive drug - Amlodipine $5 \mathrm{mg}$ daily and antiplatelet regimen. During follow-up, 24 hours ambulatory blood pressure monitoring showed good control of blood pressure with 128/75 mmHg overall average.

After 3 months no clinical or biologic signs of renal damage were noticed. Normal velocities in renal arteries

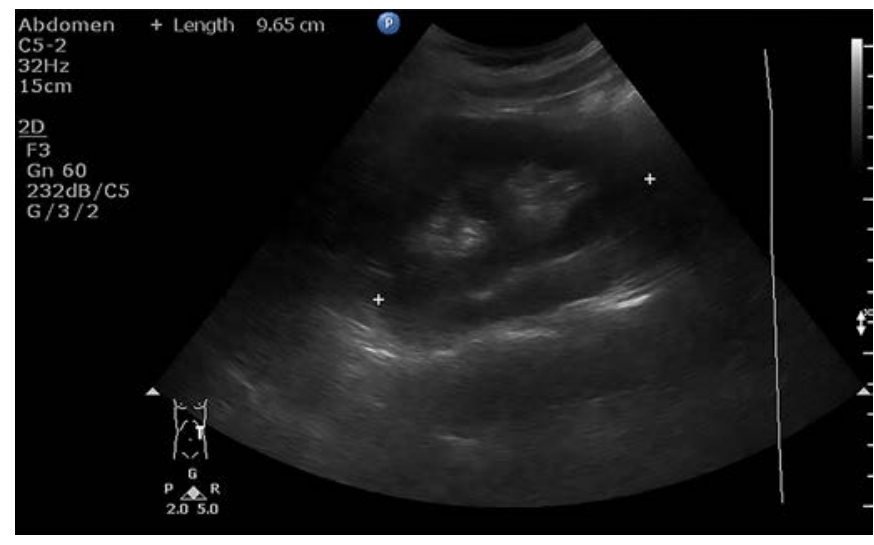

Fig. 3. Left kidney morphology in grey scale; compared to right kidney notice a long-axis difference between the kidneys.

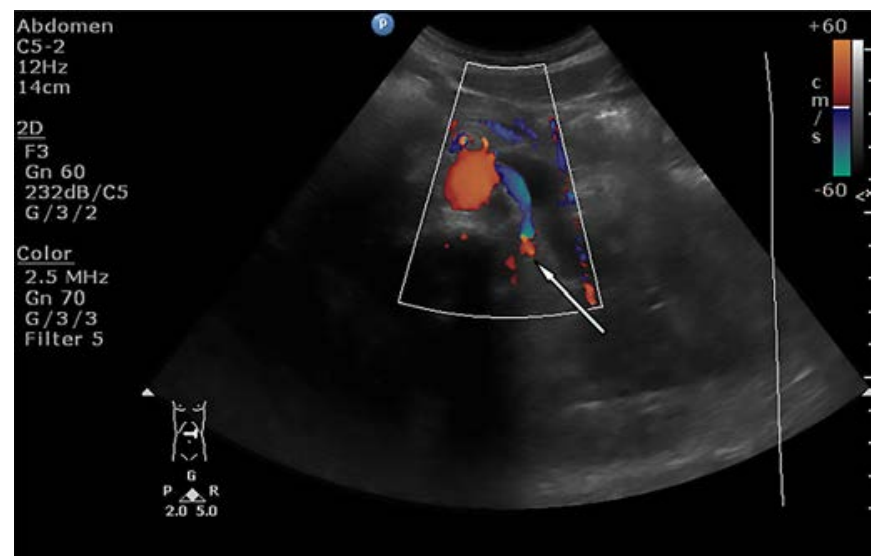

Fig. 4. Turbulent flow in the renal arteries, after the origin from aorta (arrow). 


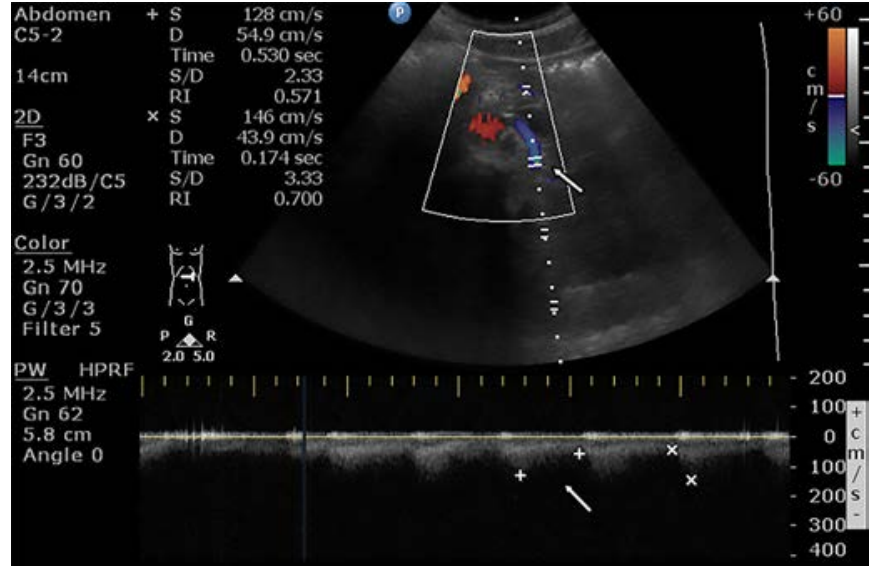

Fig. 5. Color and spectral Doppler exam (triplex Doppler) of the left renal artery. High velocities and prolonged acceleration time (arrows).

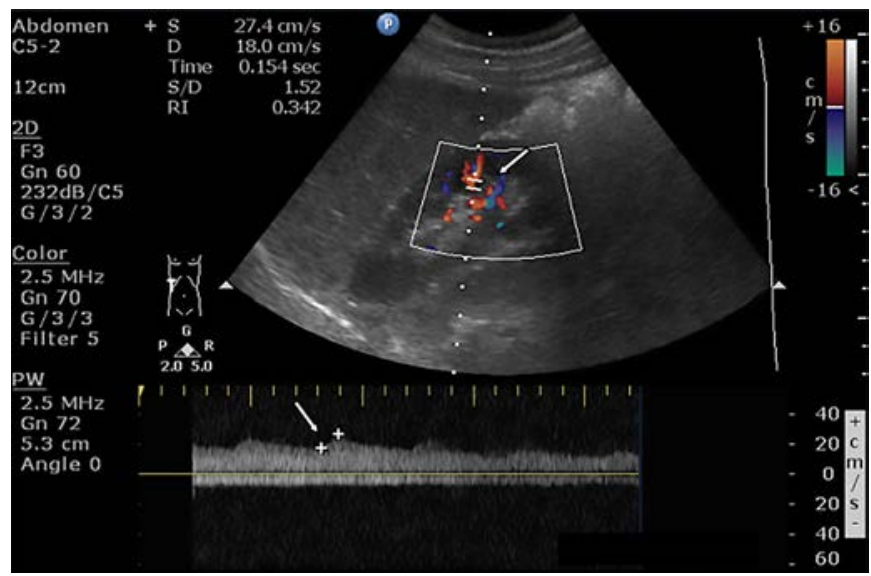

Fig. 6. Spectral Doppler waveforms in the interlobar arteries right kidney (arrows).

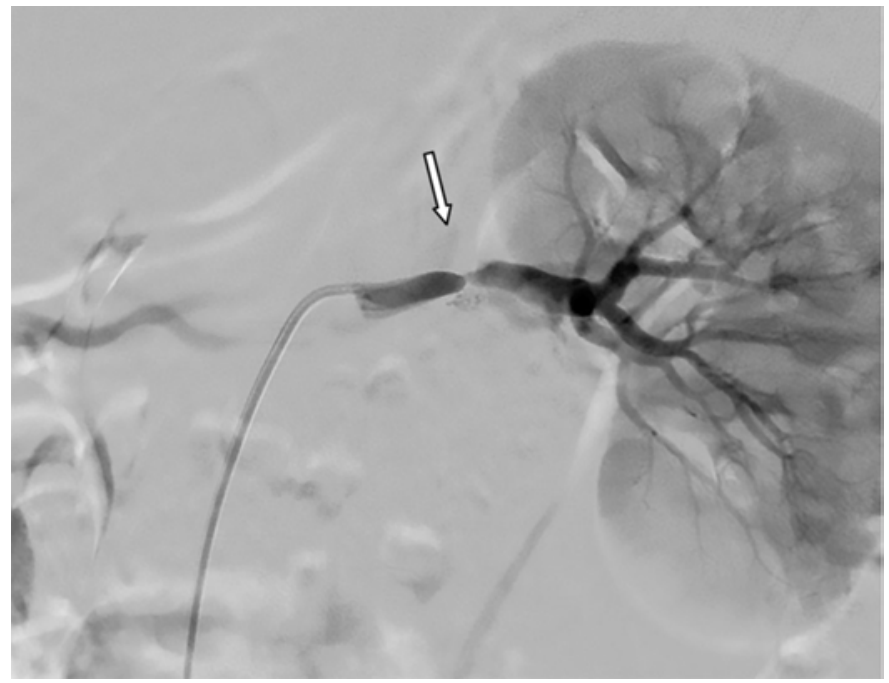

Fig. 9. Angiogram of left kidney artery (arrow).

at Doppler ultrasound examination and CT - angiogram reveals renal arteries in normal range, without involvement of cervical and brain arteries.

\section{Discussions}

The first case of fibromuscular dysplasia was reported by Leadbetter and Burkland in 1938 [8]. Genetic, hormonal

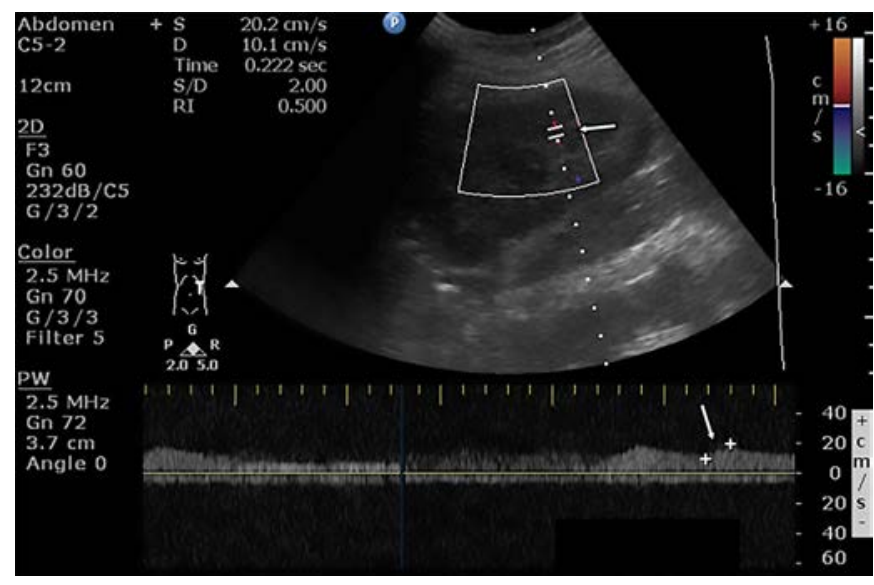

Fig. 7. Spectral Doppler waveforms in the interlobar arteries of the left kidney (arrows).

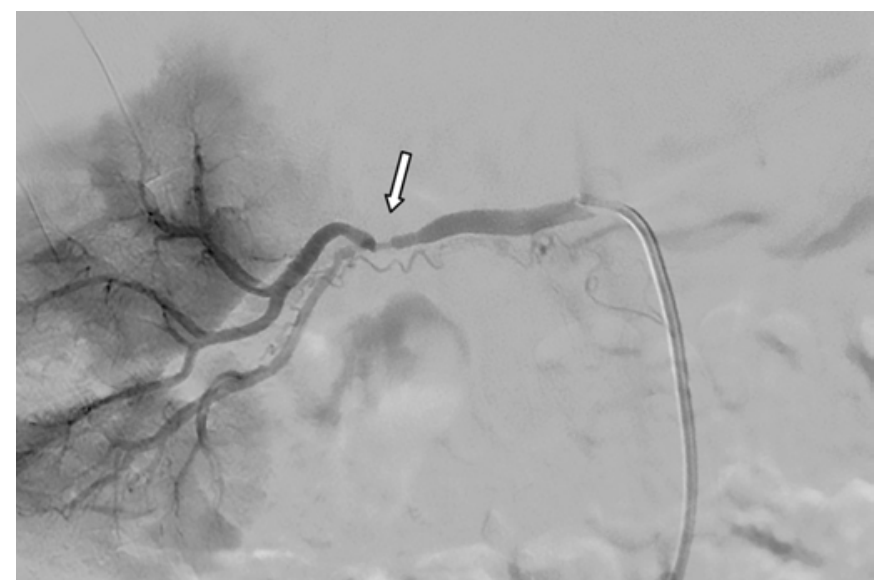

Fig. 8. Right renal artery angiogram with typical string of beads appearance (arrow)

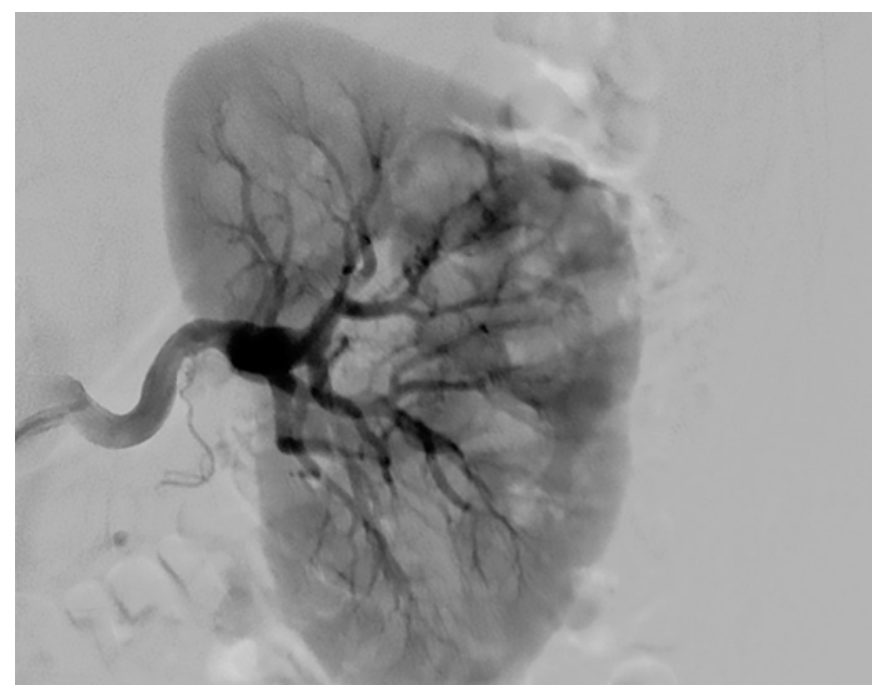

Fig. 10. Left renal artery after successful PTRA

or environmental factors are being suggested as the pathogenesis of this disease.

Abrupt onset or uncontrolled hypertension is most commonly detected in patients with renovascular hypertension due to renal artery stenosis. It is the most common clinical presentation of fibromuscular dysplasia in young woman.

Screening for fibromuscular dysplasia is recommended especially in women age bellow 30 years in cases of hyper- 


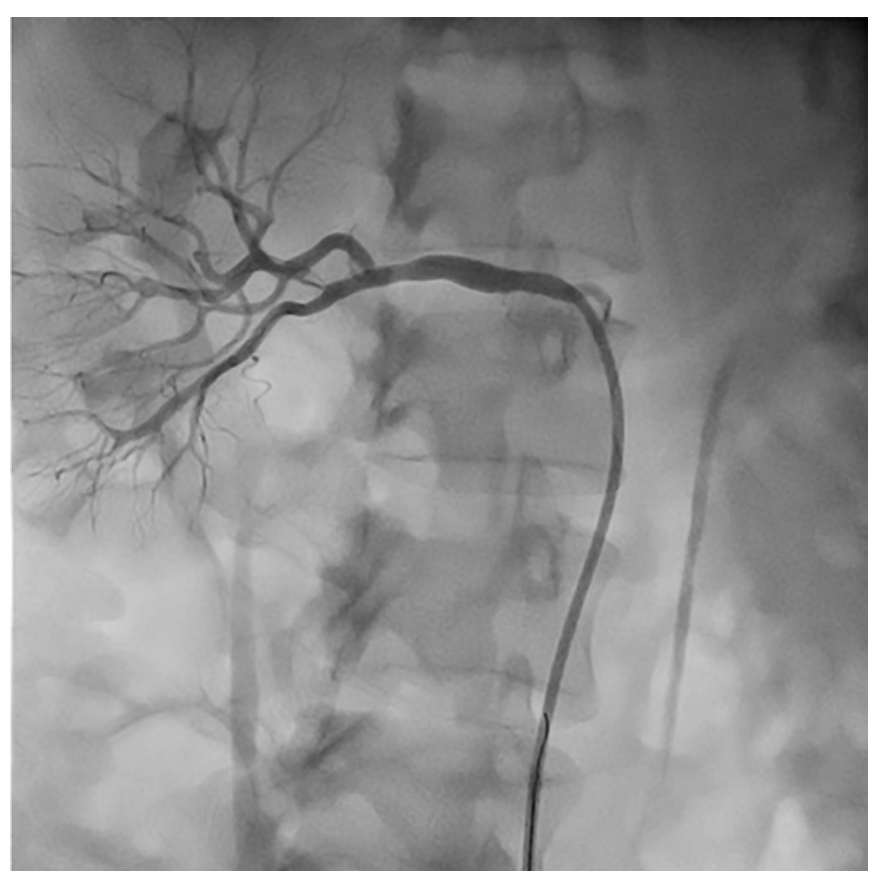

Fig. 11. Selective right renal angiogram after PTRA

tension grade $3(180 / 110 \mathrm{mmHg})$, small kidney without history of uropathy with resistant hypertension. The presence of fibromuscular dysplasia in at least another vascular territory it is a strong indication for screening [2].

Doppler ultrasound is the main non-invasive clinical investigation in a young person with hypertension when a renal artery stenosis is suspected. Renal artery stenosis is responsible for $1-10 \%$ from 50 million hypertensive patients in USA, and 5-18\% in Europe [9-11]. In our experience, the incidence was $0.7 \%$ from 3000 suspected secondary hypertension cases examined in 5 years. Most cases were older patients with atherosclerosis, only $10 \%$ of cases were RAS in young people, with renal artery fibromuscular dysplasia.

According to guideline indications for the management of blood pressure in chronic kidney disease, a moderate suspicion of renovascular hypertension based on clinical signs, ultrasound, computed tomography and magnetic resonance imaging requires isotope scans and angiographic examination. [12].

Currently, there are no randomized trials showing a benefit from intervening in fibromuscular dysplasia versus medical management.

Percutaneous transluminal renal angioplasty (PTRA) should be considered in young patients as intention to cure" the hypertension and in those who have a low renal volume secondary to ischemic nephropathy at ultrasound examination, with a success rate between $83-100 \%$ [1315].

According to published data restenosis of renal arteries after PTRA can occur in 6.7 to $23 \%$ of cases after PTRA [13-15]. Prolonged duration of hypertension and FMD involvement of branch arteries negatively are negative factors which affect the treatment [16]. Young patients with mild hypertension, a short duration of hypertension $(<8$ years), unaffected kidney function (normal serum creatinine, eGFR) seems to be good predictors in efficacy of treatment.

\section{Conclusions}

In this case we point out one important cause of secondary hypertension, fibromuscular dysplasia, and emphasizes the necessity to asses for secondary causes of hypertension in a young woman with uncommon cause of cephalalgia.

It is a good clinical practice to check blood pressure in every single young patient seen in a medical office for whatever reason. In this case use of home blood pressure monitoring supports the notion of the prognostic value of home blood pressure monitoring and helped us for the correct management of a hypertensive patient.

In the 18 years old young woman, we described first the morphology of both kidneys in grey scale ultrasound and afterward we performed a standard Doppler examination. Digital subtraction angiography was the "gold standard" for diagnosis and was followed by a successful interventional procedure.

The percutaneous transluminal angioplasty showed significant improvement in reduction of antihypertensive treatment in a young patient with secondary hypertension. Screening for asymptomatic fibromuscular dysplasia affecting other vascular territories was conducted. Brain MRI and carotid ultrasound, was negative in detecting other fibromuscular dysplasia lesions.

Medical treatment is the first indication in hypertensive patient. Further monitoring for the management of patient with fibromuscular dysplasia include blood pressure measurements at 3-month and renal function measurements annually. Non-invasive duplex ultrasonography at 12-month intervals, follow-up is indefinite. It remains to be follow-up whether the patient can be medically managed on antihypertensive medication alone.

\section{Conflicts of interest}

The authors declare no conflict of interest.

\section{References}

1. K Fendrich, M Vennemann, V Pfaffenrath, et al, Headache Prevalence Among Adolescents -The German DMKG Headache Study. Cephalalgia, 2007;27:347-354.

2. Alexandre Persua, Alessandra Giavarinic, Emmanuel Touze, et al on behalf of the ESH Working Group 'Hypertension and the Kidney', European consensus on the diagnosis and management of fibromuscular dysplasia. J Hypertens, 2014;32:1367-1378.

3. Herregods N, Beckers R, Van Rattinghe R, Verstraete K. Fibromuscular dysplasia of the carotid artery. JBR-BTR, 2008;91:195-197.

4. Touzé E, Oppenheim C, Trystram D, Nokam G, Pasquini M, Alamowitch $\mathrm{S}$, et al. Fibromuscular dysplasia of cervical and intracranial arteries. Int J Stroke, 2010;5:296-305.

5. Saw J, Aymong E, Sedlak T, Buller CE, et al. Spontaneous coronary artery dissection: association with predisposing arteriopathies and 
precipitating stressors and cardiovascular outcomes. Circ Cardiovasc Interv, 2014;7:645-655

6. Chehab BM, Gupta K. Contemporary diagnosis of carotid fibromuscular dysplasia: role of power Doppler and a review of other diagnostic modalities. Rev Cardiovasc Med, 2013;14:e136-43.

7. Johnson HM, Thorpe CT, Bartels CM, et al. Undiagnosed hypertension among young adults with regular primary care use. J Hypertens, 2014;32:65-74

8. Leadbetter WF, Burkland CE:Hypertension in unilateral renal disease. $J$ Urol, 1938;39:611-626.

9. Chrysochou C, Kalra PA. Current Management of Atherosclerotic Renovascular Disease - What Have We Learned from ASTRAL?. Nephron Clin Pract, 2010;115:c73-c8.

10. Plouin PF, Bax L. Diagnosis and treatment of renal artery stenosis. Nat Rev Nephrol, 2010; 6:151-159.

11. Jacobine M. Prevalence of atherosclerotic renal artery stenosis in patients starting dialysis, Nephrol Dial Transplant, 2003;18:1147-1151.
12. KDIGO clinical practice guideline for the management of blood pressure in chronic kidney disease. Kidney Int Suppl, 2012;2:337-414.

13. Davies MG, Saad WE, Peden EK, et al. The long-term outcomes of percutaneous therapy for renal artery fibromuscular dysplasia. J Vasc Surg, 2008;48:865-871.

14. Birrer M, Do DD, Mahler F, et al. Treatment of renal artery fibromuscular dysplasia with balloon angioplasty: a prospective follow-up study. Eur J Vasc Endovasc Surg, 2002;23:146-152.

15. Jensen G, Zachrisson BF, Delin K, et al. Treatment of renovascular hypertension: one year results of renal angioplasty. Kidney Int, 1995;48:1936-1945.

16. Alhadad A, Mattiasson I, Ivancev K, Gottsäter A, Lindblad B: Revascularisation of renal artery stenosis caused by fibromuscular dysplasia: effects on blood pressure during 7-year follow-up are influenced by duration of hypertension and branch artery stenosis. J Hum Hypertens, 2005;19:761-767. 